\title{
On the epigenetics of vascular regulation and disease
}

\author{
Christina Schleithoff ${ }^{1}$, Susanne Voelter-Mahlknecht ${ }^{2}$, Indra Navina Dahmke ${ }^{1}$ and Ulrich Mahlknecht ${ }^{1^{*}}$
}

\begin{abstract}
Consolidated knowledge is accumulating as to the role of epigenetic regulatory mechanisms in the physiology of vascular development and vascular tone as well as in the pathogenesis of cardiovascular disease. The modulation of gene expression through modification of the epigenome by structural changes of the chromatin architecture without alterations of the associated genomic DNA sequence is part of the cellular response to environmental changes. Such environmental conditions, which are finally being translated into adaptations of the cardiovascular system, also comprise pathological conditions such as atherosclerosis or myocardial infarction. This review summarizes recent findings on the epigenetics of vascular regulation and disease and presents nutritional and pharmacological approaches as novel epigenetic strategies in the prevention and treatment of cardiovascular disease.
\end{abstract}

Keywords: Epigenetics, Cardiovascular disease, Vascular regulation, Sirtuins, Histone deacetylase, HDAC

\section{Introduction}

Epigenetic alterations are chromatin-based modifications that affect the expression of genes without altering the DNA sequence itself. Such modifications include the methylation of DNA, the posttranslational modification of histone proteins and RNA-based mechanisms, [1] which may altogether modulate the tertiary structure and thus the accessibility of promoter DNA for transcription factors and numerous regulatory elements that finally affect transcription [2]. Epigenetic processes are essential as to the determination of cell identity and for the propagation of modifications that are meiotically and mitotically heritable [3]. In this context, epigenetic pathways are key elements in the regulation of endothelial gene expression, and thus in the pathogenesis of vascular disease such as atherosclerosis and vascular restenosis. The vascular system is highly flexible with regard to physiological and pathological challenges and carries therefore the potential to regenerate and generate new structures during the whole organismal life span on the basis of two major epigenetic principles: the heritable propagation of the information on changes in gene

\footnotetext{
* Correspondence: mahlknecht@k-plus.de

'Saarland University Medical Center, Department of Internal Medicine,

Division of Immunotherapy and Gene Therapy, Homburg, Saar D-66421 Germany

Full list of Author information is available at the end of the Article
}

expression without alterations in the DNA sequence, and the capacity to develop different phenotypes from one single genotype [4].

\section{Epigenetic regulation - overview}

Since Conrad Waddington first coined the term "epigenetics" back in 1942 as the study about "the causal interactions between genes and their products, which bring the phenotype into being", research progress since then led to an advanced definition [5]. More recently, epigenetics was redefined as the study of "stable alterations in gene expression without alterations in the genetic code itself" [6]. These alterations in gene expression are achieved by changes in the tertiary structure of the DNA strand and thus the accessibility of the DNA for molecules which effect gene expression. Under physiological conditions this machinery allows relatively fast heritable changes in gene expression in response to environmental factors such as nutrition or lifestyle conditions and plays a pivotal role in embryogenesis and genomic imprinting. Accordingly, the addition of activating or repressive epigenetic marks affects gene expression. The permanent silencing of one X-chromosome in women as an example is explained by heavy genomic DNA hypermethylation of CpG islands [7]. Significant alterations of the epigenome may be found in numerous diseases such as asthma or

\section{Biomed Central}


cardiovascular disease and different types of cancer, which highlights the potential of epigenetic modifications on the development of novel epigenetic treatment strategies.

The tertiary DNA structure is composed of chromatin, a complex of DNA, histones and other chromosomal proteins. The nucleosome is the fundamental component of chromatin and consists of a protein octamer that contains two copies of each of the histone proteins $\mathrm{H} 2 \mathrm{~A}, \mathrm{H} 2 \mathrm{~B}, \mathrm{H} 3$ and $\mathrm{H} 4$ with and 146 base pairs of DNA, which are being wound around this protein core [2]. Such a fibre of sequentially arrayed nucleosomes and linker histone proteins is preferentially arranged into a stable $30 \mathrm{~nm}$ solenoid tertiary structure, which is itself the basis for stable higher order chromatin compaction [8]. The degree of chromatin condensation is reversibly regulated by epigenetic mechanisms and thus, chromatin may principally be found in two variants: euchromatin and heterochromatin. Euchromatin stands for decondensed chromatin, i.e. transcriptionally active chromatin. Heterochromatin on the other hand, describes highly condensed and therefore transcriptionally inactive chromatin [2] Activating epigenetic marks are therefore predominantly found on euchromatin, while heterochromatin is primarily associated with repressive epigenetic marks [9].

\section{DNA methylation}

DNA methylation is one of the fundamental epigenetic marks that is associated with transcriptional silencing, and plays a key role not only in X-chromosomal inactivation, but also in embryonic development, genomic imprinting, and lineage specification $[1,10,11]$. In mammals, DNA methylation takes place mostly within CpG dinucleotides, where a methyl group that is derived from S-adenosyl-L-methionine is bound in position 5 within the cytosine ring, thus forming 5 -methyl-cytosine $[4,11]$. This pyrimidine can still constitute a base pair with guanine [1]. In healthy somatic cells, up to $90 \%$ of CpG dinucleotides, i.e. $3 \%$ to $6 \%$ of all cytosines, are methylated, except for promoter $\mathrm{CpG}$ islands, which appear to be protected from methylation $[7,11]$.

So far, three active DNA methyltransferases (DNMTs), which catalyse the methylation of mammalian DNA cytosines in position C5, have been described: DNMT1, DNMT3a, and DNMT3b [1] DNMT3L which belongs to the group of DNMT proteins lacks the catalytic activity itself, but is required for the enzymatic activities of DNMT3A and DNMT3B [12] DNMT2 is a highly conserved protein and shows a strong tRNA methyltransferase activity. It is known to methylate aspartic acid transfer RNA without being restricted to this process [13]. Amino acid methylation of arginine and lysine residues of proteins is usually carried out by peptilarginine or lysine methyltransferases, respectively. During embryonic development, DNMT3a and DNMT3b are responsible for de novo methylation, and for the creation of specific DNA methylation patterns [11]. While DNMT3a is essential in the context of genomic imprinting during gametogenesis, [14-16]. DNMT3b plays an important role during embryonic development [15]. DNMT1 is required for the maintenance and propagation of DNA methylation patterns across generations of cells, i.e. the methylation pattern is carried along the replication process during mitotic cell division [1]. DNMT1 is however not that efficient as to the maintenance of methylation patterns within CpG dense regions [11] and during massive demethylation events such as after fertilization, [14] which may as a consequence lead to alterations of the original methylation pattern. Three principal mechanisms are involved in the regulation of gene repression via 5-methylcytosine: First, 5-methyl-cytosine can sterically affect transcription factors to their cis-DNA binding elements, as it is described for several transcription factors [17-20]. During this process, methyl groups of methylated $\mathrm{CpG}$ dinucleotides intercalate into the major groove of the DNA helix [1]. Second, methyl-CpG binding proteins such as MeCP2, interfere with the recruitment of transcription factors (i.e. DNA-binding trans factors) [2]. Third, these methyl-CpG binding proteins are able to recruit large protein complexes which control the accessibility of DNA through modification of the chromatin structure [11]. On the other hand, a transcriptional activator, human $\mathrm{CpG}$ binding protein (hCGBP), has been reported that specifically recognizes and binds unmethylated GpG dinucleotides [21]. Even though, both, active (replication-independent), as well as passive (replication-dependent) mechanisms have been described, the process as of how DNA methylation marks are exactly removed, is still unexplained [11]. Therapeutically, nucleoside analogues require proliferating cells in order to inhibit DNA methylation, because the nucleoside analogues exert their activity through an irreversible covalent bond with DNMTs after their incorporation into DNA [7].

\section{Histone proteins}

The histone proteins within the nucleosomal core consist of a globular domain and an N-terminal tail, which may be subjected to more than 60 variations of posttranslational modification [2] .Lysine for example, can be modified by acetylation, methylation, ubiquitylation or sumoylation, while arginine can be modified solely by methylation; and finally serine and threonine by phosphorylation $[10,22]$. In contrast to DNA methylation, most histone posttranslational modifications are highly dynamic processes [1]. Even though some of these covalent modifications take place within the histone globular domains, the best studied modifications - and more specifically the effects of lysine acetylation and methylation on chromatin condensation and thus on the regulation 
of the activity of gene promoters, are within the histone $\mathrm{N}$-terminal tails $[10,22,23]$.

\section{Histone acetylation}

Histone acetylation goes along with transcriptional activation and is mediated by histone acetyltransferases (HATs), $[1,2,24]$ while it is antagonized by the opposing histone deacetylase (HDAC) enzymatic activity, which mediates the removal of acetyl groups and therefore goes along with chromatin compaction and transcriptional inactivation. HDACs are grouped into four classes according to their relationship to their homologues in S. cerevisiae: class I (HDAC1-3, HDAC8), class II (HDAC4-7, HDAC 9-10), class III sirtuins (SIRT1-7), and class IV (HDAC11) [22]. With the exception of class III HDACs - sirtuins, which are $\mathrm{NAD}^{+}$-dependent, all other HDACs are $\mathrm{Zn}^{2+}$ dependent. Both, HATs and HDACs unspecifically influence the acetylation status of proteins. Their specificity as to the posttranslational modification of histone proteins may - at least in part - be achieved through their recruitment to chromatin within multi-protein complexes, [22] and their localization within specific cellular compartments. Massive acetylation of lysine residues may activate transcription through neutralization of the basic charge of these residues and through the recruitment of bromodomain-containing protein complexes, which may include other HATs and chromatin remodeling enzymes [25]. This process goes along with an easily accessible chromatin configuration, so that transcription is facilitated [26]. The acetyl groups that are needed for this process are transferred from acetyl-coenzyme A complexes, and the reaction is catalyzed by three principal families of HATs: CBP/ p300, GNAT and MYST [1]. The different families of mammalian HDACs are grouped in four classes: class I (HDAC1-3, HDAC8), class II (HDAC4-7, HDAC 9-10), class III sirtuins (SIRT1-7), and class IV (HDAC11) $[22,27,28]$.

\section{Histone methylation}

Unlike the acetylation of histone proteins, the methylation of histone lysine and/or arginine residues has variable effects on gene expression. The methylation of histone lysine residues has been extensively studied and in fact, the effect on gene expression very much depends on the specific lysine residue that is being modified. Also, the single lysine residues can be variably methylated to mono-, diand trimethylated states $[2,29,30]$. Every such status is essential and allows a highly specific distinction concerning the methylation pattern: active promoters for example, are enriched in trimethylated H3 lysine 4 (H3K4) residues, whereas enhancer elements are enriched in monomethylated H3K4 residues [31]. On the other hand, di- and trimethylated histone $\mathrm{H} 3$ lysine 9 (H3K9) residues are strongly correlated with transcriptional repression [22,32].
Enzymes that catalyze this reaction, act dynamically as either histone methyltransferases or histone demethylases. e. g. lysine-specific histone demethylase 1 which demethylases $\mathrm{H} 3 \mathrm{~K} 4$ or Jumonji C (JmjC)-domain-containing family of proteins which catalyse demethylation of H3K4me3 [29]. Few lysine residues can be either methylated or modified by acetylation, but never both together [2].

\section{RNA-based mechanisms}

So far, the RNA-based mechanisms are the least well understood mechanisms of epigenetic regulation. Noncoding RNA (ncRNA) appear to be involved in the chromatin-based regulation of gene expression [2,6,33]. In fact there is increasing evidence that RNA-based mechanisms combine the coordinated activities of this noncoding RNA together with other epigenetic modifications, such as DNA methylation and posttranslational histone modifications. For example the RNA-induced initiation of transcriptional gene silencing complex (SISC) forms heterochromatic sites as a consequence of histone methylation[34]. Similarly, the expression of ncRNA itself may be modulated through epigenetic mechanisms: miRNA 124 for instance, is silenced in different types of cancer by DNA-methylation. These are only two examples for the complexity and intertwinement of epigenetic mechanisms. NcRNAs are defined by their number of nucleotides: Short ncRNAs count up to 200 nucleotides long ncRNAs count more than 200 nucleotides [2]. These ncRNAs seem to regulate the chromatin compaction state of defined genomic loci [35]. Large intervening non-coding RNAs (lincRNAs) are able to recruit chromatin modifying complexes and/or to overlap the coding region of genes and thus to regulate gene expression at the level of specific target loci $[2,36]$. As an example, Khalil et al. found about $20 \%$ of 3,300 human long intergenic ncRNAs were bound by Polycomb Repressive Complex 2 (PRC2) or other chromatinmodifying complexes [36]. In addition, long ncRNAs ( $>200$ nucleotides) are able to mediate transcriptional activation by recruiting the H3K4 mixed-lineage leukemia methyltransferase (MLL1) [35]. Small non-coding RNAs on the other hand, are $\sim 21$ bp long with target messengers that can induce degradation of the dsRNA complex through the action of the RNA III endonuclease (also referred to as "Dicer") [37]. The transcriptional silencing by small ncRNAs like microRNAs (miRNA) and short interfering RNAs (siRNA) is currently being investigated very intensively due to their promising therapeutic potential. These small RNAs are grouped into different classes depending on their length and function [38]. They are able to specifically inhibit target mRNAs of different genes. MiRNAs and siRNAs are 21 to 26 nucleotides long, and are well-known mediators of tissue-specific posttranscriptional gene silencing as a part 
of RNA-induced silencing complex (RISC) and as siSC (scrambled inhibitory RNA) respectively [1,37] miRNAs are synthesized by RNA polymerase II as a long RNA primary transcript (pri-miRNA) and cleaved by Drosha, the double-stranded RNA endonuclease III, to produce a typical stem-loop structure, known as a pre-miRNA [39]. The nuclear RNase III Drosha initiates microRNA processing [39]. Drosha is part of a multi-protein complex, which contains the double-stranded RNA binding protein Pasha also known as DGCR8 [40]. Pasha binds single-stranded pri-miRNA fragments and stabilizes them for processing by Drosha. Additional enzymes that promote the maturation of miRNA are two proteins called Argonaute and Dicer which are part of RISC. Drosha and Pasha are both localized within the cell nucleus, where the processing of pri-miRNA to pre-miRNA takes place. This pre-miRNA is then exported into the cytoplasm via Exportin 5 and then further processed by the RNase Dicer to dsRNA which is then transported back into the nucleus via RISC. The mature single strand ('guide strand') is paired to the 3'-untranslated region of the target RNA and leads to its degradation [41]. SiRNAs are derived from long double-stranded RNA precursors and may be found in cells naturally or the may be exogenously imported and in fact, transcriptional gene silencing can be achieved by exogenously administered siRNAs directed to promoter regions in mammalian cells [1]. The mechanisms that are involved herewith are sitespecific DNA methylation [42] and repressive histone posttranslational modifications [43]. In the future, studies might demonstrate the far-reaching effects of RNAbased mechanisms on the regulation of mammalian gene expression.

\section{Epigenetics of blood vessel physiology}

During development and lifelong sustainment of the vascular system epigenetic factors play a crucial role and permit a high flexibility to sudden physiological changes.

\section{Epigenetic mechanisms in vascularization}

After fertilization, the vascular system is one of the first organ structures that develop. During "vasculogenesis" a primary capillary plexus is formed de novo from mesodermal cell precursors, while the formation of new vessels from pre-existing vessels is referred to as "angiogenesis" and is based on the interaction of pro- and antiangiogenic molecules [44]. The initiation of vascular development depends on the presence of Fibroblast growth factors in order to induce hemangioblastic differention. In a next step vascular endothelial growth factor (VEGF) further triggers cell differentiation. The formation of a capillary plexus strongly depends on the expression of adhesion molecules which form inter-cellular connections such as VE (vascular endothelial) cadherin, N-cadherin and connexins, as well as molecules which promote cell matrix interactions (netrins, semaphorins, fibronectin, integrins) and a number of signaling pathways that include key proteins such as NOTCH, VEGF1/2, transforming growth factor beta (TGF-ß), or ephrin type-A receptor 2 (Eph-2) and 4 , only to mention a few $[45,46]$. The acetylation of Notch via SIRT1 regulates the amplitude and duration of Notch activity and allows for normal vascular sprouting [47]. In a second stage, haemodynamic factors and local hypoxia model the structure, identity and function of the blood vessels, [48-50] which accentuates the role of epigenetic mechanisms [4]. In summary, epigenetic mechanisms strongly influence the development of the vascular system $[1,51]$.

Vascular development, as well as endothelial and smooth muscle cell differentiation and function, require a fine epigenetic adjustment. The promoter regions of several cell-specific endothelial proteins such as the endothelial nitric oxide synthase (endothelial isoform of NO synthase, eNOS [alias: NOS3]) are constitutively active [52] and it appears that the key difference to other cell populations that do not express eNOS is observed at the chromatin level: while a relaxed and thus transcriptionally permissive chromatin structure may be found at the level of the eNOS promoter in endothelial cells, this very locus appears to be highly condensed and thus in a repressive configuration in non-endothelial cells [2]. In addition, the expression of the human eNOS-gene is at least in part, also determined by the DNA methylation status [53] Matouk et al. proposed that additional chromatin-based mechanisms could be relevant for the cellspecific expression of eNOS. In non-expressing cell types, the eNOS promoter was hypermethylated and lacked activating histone posttranslational modifications, whereas this very promoter was reported to be hypomethylated and enriched with activating posttranslational histone modifications such as acetylated H3K9, H4K12 as well as di- and trimethylated H3K4 in endothelial cells $[53,54]$. Endothelial-specific transcription factors such as KLF2 are also involved in the regulation of the tissue specific expression of endothelial proteins, [55] moreover, KLF2 is not even responsible for the differentiation of precursor cells to endothelium which underlines the significance of epigenetic regulation [4].

Several studies show that HDAC activity mediates angiogenesis as a consequence of hypoxia, [56] shear stress- and VEGF-induced stem cell differentiation $[57,58]$. On the other hand the inhibition of HDAC prevents the proliferative response to VEGF [59]. There is some evidence that the global and endothelial cellspecific knockout of HDAC 7, a class II HDAC, is associated with embryonic lethality and required for the development of a normal vasculature [60]. HDAC7 is an important effector molecule in the context of the VEGF- 
signaling pathway: the suppression of HDAC7 phosphorylation and subsequent translocation to the cytoplasm inhibits VEGF-mediated proliferation and migration [61]. HDAC activity is known to be critical for the endothelial differentiation of embryonic stem cells as well as adult endothelial progenitor cells $[57,58,62,63]$. Histone acetylation controls the expression of important signaling molecules like the von-Willebrandt-factor, NOTCH and eNOS [52]. Nevertheless, for the sprouting process during angiogenesis, histone methylation appears to be very important [64].

Several studies correlated environmental stimuli during development with non-Mendelian diseases, such as cardiovascular disease. The persistence and reproducibility of these studies indicate permanent non-genomic modifications, which result in a certain cellular "re-programming". The modulations needed for this might be based and - at least in part - explained by epigenetic mechanisms [4]. Such studies show that epigenetic modulations also reflect environmental signals under physiological and pathological conditions $[65,66]$. However, only little information is available on the varying response in foetal endothelial cells in the context of different pregnancy related diseases $[67,68]$. Important evidence for the immediate role of epigenetic mechanisms in pathological alterations is found in diabetes. In this context, it has already been shown how intra uterine growth restriction (IUGR) generates abnormal programming of gene expression at different levels, which could advance the development of insulin resistance and/or type 2 diabetes [4].

\section{Postnatal angiogenesis}

During the postnatal period, HDACs have been reported to be key regulators of angiogenesis [1]. Accordingly, HDAC inhibitors have already proven to exhibit potent antiangiogenic activity [69]. Some HDACs appear to have prominent roles in this context: HDAC1 for example plays a pivotal role in hypoxia-induced angiogenesis [70]. On the other hand, specific knockdown of HDAC7 constrains cell migration and angiogenesis in mature primary endothelial cells in culture [71]. Another important factor in angiogenic signaling is constituted by SIRT1, a class III HDAC [72]. SIRT1 is highly expressed during blood vessel growth and allows for sprouting during angiogenesis through deacetylation of the forkhead transcription factor Foxo1 [73].

So far, little information is available on what is going on within the specific angiogenesis pathways, but it is likely that the acetylation status of various transcription factors that are being involved plays an important role [1].

\section{Shear stress}

Laminar flow may influence gene regulation via epigenetic pathways and thus, disturbed flow is capable of changing gene expression in cells [2]. Shear stress for instance, enhances histone H3 K79 methylation in mouse embryonic stem cells (ESC), [58] where shear stress causes global histone modification changes and promotes ESC differentiation to an endothelial cell lineage $[58,74]$. Accordingly, laminar shear stress also goes along with both, global and gene-specific histone modifications in cultured human endothelial cells [75]. Shear stress enhances the activity of HAT p300 in human endothelial cells and leads to the acetylation of $\mathrm{H} 3$ and $\mathrm{H} 4$ at the level of the eNOS promoter among others [30] HDACs can also modify gene expression in response to shear stress for example through the VEGF-signaling pathway: Zeng et al. showed that HDAC3-mediated p53 deacetylation and p21 activation caused by shear stress and VEGF induces differentiation of endothelial cells through the VEGF receptor 2 (Flk-1)-PI3K-Akt signal pathway [57].

\section{Hypoxia}

Intrauterine hypoxia i.e. lower oxygen tension in the fetus compared to the adult, is to a certain degree essential for cardiac formation. Hypoxia decreases global gene expression via epigenetic pathways: A global decrease in H3K9 acetylation can be observed in various types of cells as a consequence of increased HDAC activity $[76,77]$. On the other hand, acetylated H3K9 is found at the promoter level for hypoxia activated genes such as VEGF [76-78]. Increasing evidence also accumulates that the hypoxia- inducible factor (HIF-1 $\alpha$ ) is involved in the maintenance of global transcriptional silencing, as well as in directing gene repression to specific genes $[79,80]$ Kato et al. showed that HDAC7 is transported to the nucleus together with HIF- $1 \alpha$ under hypoxic conditions where it upregulates the transcriptional activity of HIF$1 \alpha$ forming a complex together with p300 [80]. In conclusion, the current understanding of hypoxia-induced epigenetic changes is comparatively poor and needs further investigation.

\section{Cardiovascular disease}

In the past few years, there has been increasing evidence that part of the gene-environmental interactions is regulated by epigenetic mechanisms. Accordingly, abnormal regulation in this context can result in complex diseases.

\section{Atherosclerosis and restenosis}

Some of the genes that are regulated by epigenetic modification constitute a major part of the regulatory work of extracellular matrix formation, inflammation and proliferation, which are involved in cardiovascular pathology including atherosclerosis and restenosis [3]. Both, atherosclerosis and restenosis are intensively affected by the inflammatory response to endothelial injury and the subsequent reshaping of the vessel wall in size and composition, also referred to 
as "remodelling" (Figure 1) [81,82]. For both disorders the proliferation and migration of vascular smooth muscle cells (VSMCs) and the formation of extracellular matrix, leads to an accumulation of collagen and proteoglycans, which finally results in to the occlusion of blood vessels [3]. For atherosclerosis, elevated lipoprotein levels and cigarette smoke are a main effectors, [83] while restenosis is mainly an overshooting wound healing process in response to vascular injury by balloon dilation or stent placement. Atherosclerosis develops from the accumulation of oxidized lipoproteins within foam cells and extracellularly together with the proliferation of arterial smooth muscle cells. The incidence of atherosclerosis is associated with increasing age, whereas restenosis is develops rapidly after interventions of revascularization [3] Global DNA hypermethylation appears to be significantly associated with vascular inflammatory response to endothelial injury and correlates with cardiovascular mortality [84] The underlying mechanisms that lead to inflammation are believed to be based on the inactivation of suppressors of cytokine signaling (SOCS) [85]. Atherosclerosis and restenosis are worsened as a consequence of multiple inflammatory pathways, which are regulated by pro-inflammatory transcription factors like NFK-B $[3,86]$. The activity of $\mathrm{NF} \kappa \mathrm{B}$ is known to be regulated through posttranslational acetylation, i.e. by HATs and HDACs [87]. Eotaxin, which is a chemoattractant for eosinophilic granulocytes, is an important player in the context of inflammatory response and appears to be a target of epigenetic regulation: inflammatory signaling via TNF- $\alpha$ specifically leads to histone $\mathrm{H} 4$ acetylation and induces the binding of p 65 to the eotaxin promoter, which induces transcription of the eotaxin gene and consequently, increasing numbers of inflammatory cells are being recruited and activated within the atheromatous plaque (Figure 2) $[88,89]$. Another factor that plays an important role in atherosclerosis and restenosis is the granulocyte macrophage colony-stimulating factor (GM-CSF). In a rabbit model, GM-CSF injections have been shown to reduce the formation of neointima and in accordance with a patient study, NFkB-mediated inflammatory signaling concurs with histone $\mathrm{H} 4$ hyperacetylation, which correlates with increased GM-CSF expression levels $[3,90]$. The predominant finding in this study was a reduction in the progression of artherosclerosis by GM-CSF.

Furthermore the HDAC inhibitor trichostatin A (TSA) increases the expression of GM-CSF in alveolar macrophages and airway epithelial cell lines after activation with inflammatory stimuli, [91-93] which suggests that the signalling pathway that involves GM-CSF could affected by alterations at the epigenomic level. Numerous other factors such as cyclooxygenase- 2 do also appear to be regulated via epigenetic modifications. Taken together, epigenetic modulation seem to play a key role in the regulation of inflammatory events and could

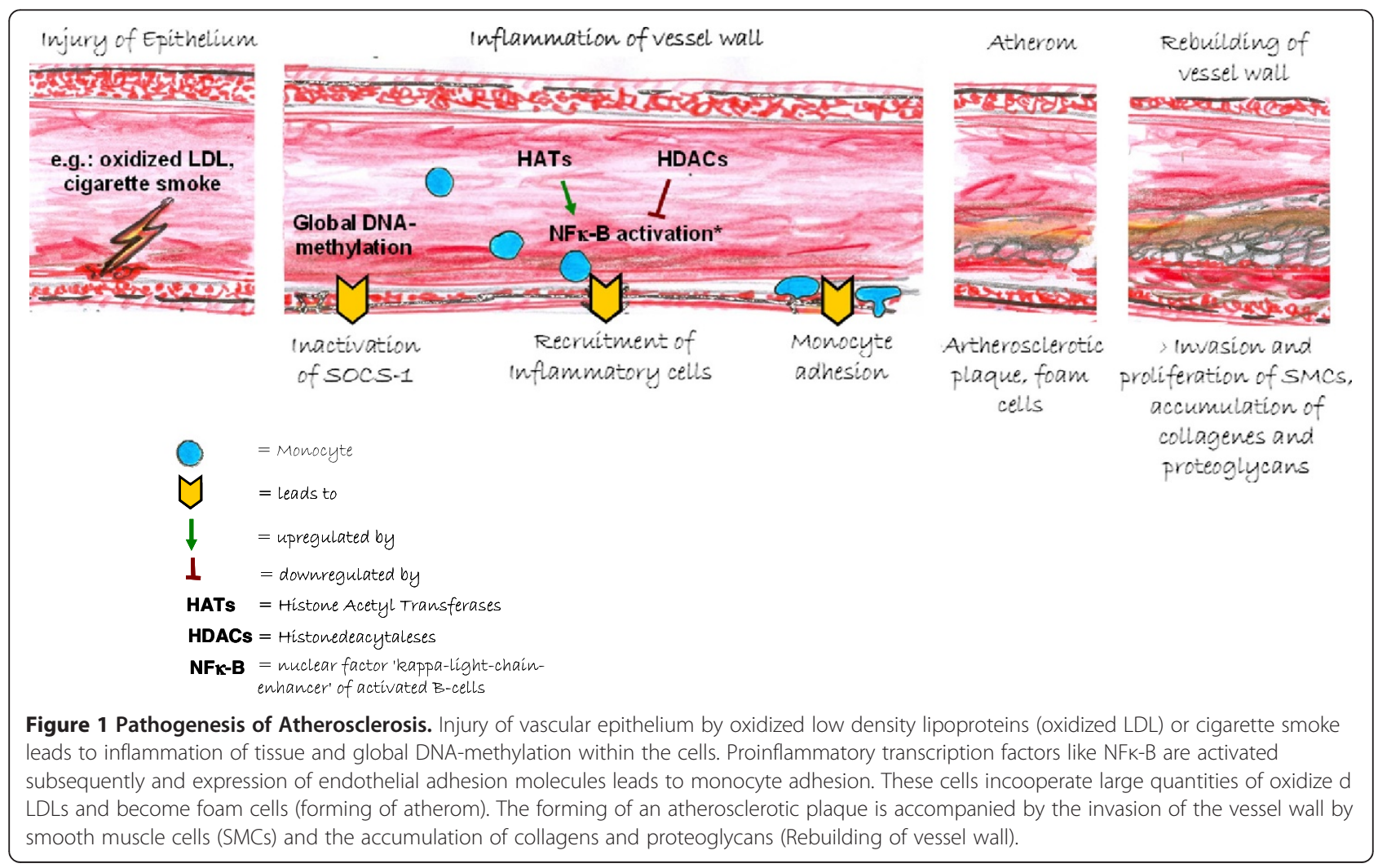




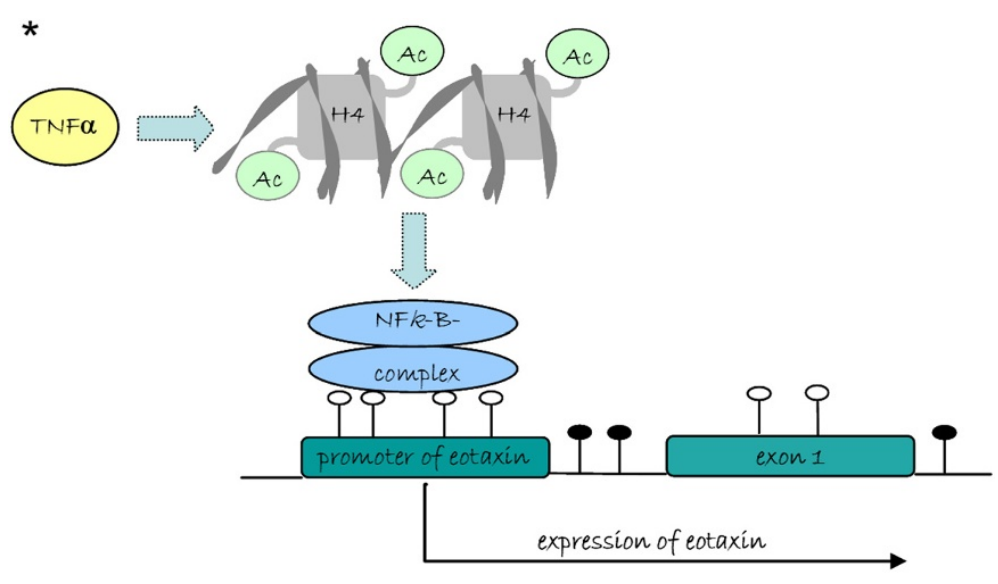

$$
\begin{array}{ll}
\uparrow & =\text { methylated cpG site } \\
9 & =\text { unmethylated cpG site } \\
\text { TNF } \alpha & =\text { Tumornecrosisfactor alpha } \\
A C & =\text { Acetylgroup } \\
H 4 & =\text { Histone H4 }
\end{array}
$$

Figure 2 Epigenetic modulation of Eotaxin expression via NFK-B. Inflammatory signalling via TNF-a leads to acetylation of histone $\mathrm{H} 4$ and induces the binding of p65 subunit of NFKB-complex to unmethylated sites of the eotaxin promoter. Transcription of the eotaxin gene is induced and consequently the number of inflammatory cells which are recruited and activated within the atherosclerotic plaque.

therefore become important structures of targeted medical therapy.

\section{Myocardial infarction}

In a murine model of ischemia and reperfusion, ischemia was shown to induce HDAC activity in the heart and consequently deacetylation of histones $\mathrm{H} 3$ and $\mathrm{H} 4$ [94]. From $1 \mathrm{~h}$ prior to ischemia until $45 \mathrm{~min}$ after reperfusion, the HDAC inhibitors TSA and scriptaid were able to reverse the activity of ischemia-induced HDACs in vivo and to reduce myocardial infarct size by up to $50 \%$. In an in vitro study, it has been shown that a $5 \mathrm{~h}$ period of hypoxia resulted in a strong decrease in acetylated $\mathrm{H} 3$ and $\mathrm{H} 4$ histones in mouse cardiomyocytes, which was completely blocked by TSA [94]. In 2002 Mc Kinsey et al. found that class IIa HDACs (i.e. HDAC4, $-5,-7$, and -9$)$ interact with members of the myocyte enhancer factor-2 (MEF2) transcription factor family which are key regulators of cardiac hypertrophy. Therefore, the down regulation of these HDACs raises new possibilities as to cardiovascular disease treatment and prevention [95] Zhang et al. demonstrated that the inhibition of histone deacetylases (HDAC) protects the heart against ischemia-reperfusion (I/R) injury in which the acetylation of NFkBp50 plays the pivotal role [96]. In summary, epigenetic pathways play an important role in myocardial infarction, and HDAC inhibitors could therefore open new horizons as to improved individualized treatment strategies in the future.

\section{Abdominal aortic aneurysm}

Abdominal aortic aneurysm (AAA) appears to be the result of an imbalance between aortic extracellular matrix destructive and restorative processes [8]. In this context, the normal lamellar architecture of the aorta gets destroyed and is subsequently invaded by inflammatory cells, including T/B lymphocytes, macrophages, neutrophils, mast cells and plasma cells [97]. In animal models, AAA have been shown to be a dynamic remodelling process, with neovascularisation, inflammatory cell infiltration, endothelial dysfunction, apoptosis and depletion of the vascular smooth muscle cells, as well as destruction of the elastic media [98]. The pathogenesis of AAA is poorly understood, and there seem to be multiple environmental and genetic factors which cause the initiation and progression of AAA. Many of the AAA risk factors including cigarette smoke, older age, male gender and hypertension, have already been linked to epigenetic effects and could promote AAA. Previous investigations demonstrated that persistent inflammation in the context of atherosclerosis might lead to aberrant DNA methylation as well as alterations in histone modification. Given that AAA has a strong inflammatory component, it is likely that epigenetic mechanisms are involved in the 
progression of AAA [8]. In human AAA biopsies, the matrix metalloproteinase-2 and 9 (MMP) whose proteolytic activity contributes to matrix degradation in AAA, are significantly upregulated and the expression of these MMPs is in fact regulated by posttranslational acetylation at the histone level $[8,99,100]$.

\section{Raynaud's syndrome}

Even more than 100 years after its first description, the pathogenesis of Raynaud's syndrome is still quite unclear $[101,102]$. The interaction of different regulatory mechanisms between endothelium, smooth muscle and autonomic and sensory innervation, which ultimately lead to the pathological vasospasm of this disease, is extremely complex and affects both, endothelium-dependent and endothelium-independent mechanisms of vasoregulation. The endothelial vasoregulation is based on the interplay between opposing vasoconstrictive mediators (e.g. plasma endothelin-1 [EDN1], thromboxane [TXA 2], methyl arginine, transcription factor HIF- $1 \alpha$ [hypoxia-inducible factor 1-alpha] and acetylcholine) and vasodilating agents (e.g. prostaglandin [prostacyclin], CGRP [calcitonin gene-related peptide] and eNOS [102-104]. The activity of eNOS - as an example for a gene that is being regulated at the epigenomic level - can be reduced by histone deacetylase (HDAC) inhibitors, which consequently results in reduced vasorelaxation [52]. Furthermore, there are direct interactions between epigenetic regulators and proteins, being involved in the regulation of the vascular tone, e.g. the transcription factor HIF- $1 \alpha$, which interacts directly with histone deacetylases [80].

Importance of $\mathrm{NO}, \mathrm{NADPH} / \mathrm{NADH}$ oxidase in the modulation of vascular tone

The vascular endothelium is not only a structural semiselective diffusion barrier between the vessel lumen and the interstitial space and contributes to the modulation of inflammation and coagulation, but also regulates blood pressure, vascular tone and blood flow by the release of a large number of vasodilating substances such as nitric oxide (NO) and prostacyclin (PGI2) and of vasoconstrictors such as endothelin (ET) and platelet factor (plateletactivating factor (PAF)) [105-107]. In addition to its role as a vasodilator, there are a number of other biologically important effects of NO: NO improves the perfusion of the coronary arteries and the cardiovascular system. It can lead to swelling of the corpus cavernosum of the penis and can relax the vascular muscle and the bronchial tree. NO is released from the endothelial cells and inhibits prostacyclin in combination with the aggregation of platelets [108]. Nitric oxide (NO) is present in almost every organ system and is produced by the endothelial NO synthase by the cleavage of the amino acid L-arginine. Because of its short half-life, it is generated directly in the target area. eNOS is expressed mainly by the vascular endothelial cells of the tunica media of the arterial blood vessels. Its production is influenced by various physiological and pathophysiological stimuli $[109,110]$. The expression of eNOS is regulated at the transcriptional and at the posttranscriptional level. Meanwhile, several physiological and pathophysiological stimuli, influencing the transcription of the eNOS gene, are known: Thus, the mRNA expression of eNOS is significantly increased in response to lysophosphatidylcholine, shear stress and TGF-beta [111]. The eNOS mRNA level not only depends on the transcriptional activity at the promoter level, [111]. the post-transcriptional modulation of the eNOS mRNA half-life is probably the determining factor that influences the actual amount of eNOS mRNA. eNOS mRNA can be stabilized through exposure of endothelial cells to 3-hydroxy-3-methylglutaryl (HMG)-CoA reductase inhibitors, [112] the VEGF or the exposure to shear stress $[113,114]$.

TNF-alpha, [115] oxLDL, [116] hypoxia, [116] and cell confluence, [116] however, accelerate the degradation of eNOS mRNA. The expression of eNOS mRNA is pathological in a variety of vascular disorders [111]. A decrease of the expression of eNOS mRNA has been described in cardiovascular diseases [54].

The half-life and biological activity of $\mathrm{NO}$ is decisively affected by reactive oxygen derivatives (ROS), such as $\mathrm{O}_{2}$ $[117,118] \mathrm{O}_{2}$ and nitric oxide (NO) are important substrates as to the regulation of vasodilation and vasoconstriction. These small molecules exhibit opposing effects on the vascular tone and result in the production of potentially toxic substances, such as peroxynitrite (ONOO) [119-125]. The endothelial bioavailability of NO in atherosclerosis and in heart failure is reduced through a decreased activity of antioxidant enzyme systems (ecSOD = extracellular superoxide dismutase) and increased activity of oxygen radical-producing enzyme systems (NADPH oxidase, xanthine oxidase). In endothelial cells and in vascular smooth muscle cells, this membrane-associated, $\mathrm{NAD}(\mathrm{P}) \mathrm{H}$-dependent oxidase is the most important $\mathrm{O}_{2}$-producing enzyme in endothelial cells, smooth muscle cells and/or vascular adventitia. There may be a link between the $\mathrm{NAD}(\mathrm{P}) \mathrm{H}$ - dependent oxidase NAD, being required by sirtuins. It is reported that the eNOS activity can be reduced by HDAC inhibitors, resulting in an impaired relaxation of blood vessels [52].

In summary, the endothelium-dependent vasoregulation depends on the interplay between opposing vasoconstrictive (e.g. plasma endothelin-1 (EDN1), thromboxane (TXA2), methyl arginine, transcription factor HIF- $1 \alpha$ and acetylcholine), and vasodilating activities (e.g. prostaglandins (prostacyclin), CGRP (calcitonin gene related peptide) and the endothelial isoform of nitric oxide synthase (eNOS, synonym: NOS3)) [102-104]. 
The role of non-coding RNAs in cardiovascular disease MicroRNAs (miRNAs) belong to the group of non-coding RNAs and work as regulators of gene expression at the mRNA level by suppression of translation and finally degradation of mRNAs for example by Ago2 mediated cleavage [38]. The expression profile of miRNAs in a rat model of vascular restenosis showed that 100 out of 140 miRNAs were differentially regulated during neointima reformation. In this context miRNA 21 was identified to be a key regulator of cell proliferation and the redifferentiation of smooth muscle cells (SMCs) [126]. Downregulation of miRNA 145 and miRNA 143 after vascular injury modulates the SMC cytoskeleton at least in part by induction of KLF5, which further reduces SMC contractility. SMC proliferation is among other things - enhanced by the downregulation of cell cycle inhibitors such as p27 and p57 and by increased endogenous levels of miRNA 221 and miRNA 222 [127]. The inflammation of vessel walls during the development of atherosclerosis is enforced by the downregulation of miRNA 126 which promotes the expression of VCAM- 1 on one hand, while it induces the production of CXCL12 on the other leading to the recruitment and adhesion of inflammatory cells $[128,129]$. As reported by Menghini et al. an increased expression of miRNA 217 in atherosclerotic plaques in patients leads to the disintegration of the endothelium and thus to the acceleration of vascular senescence via inhibition of SIRT1 [130]. Since miRNAs appear to be of utmost importance in the pathogenesis of cardiovascular disease, the profiling of circulating miRNAs might be a useful biomarker for disease assessment [131]. Fichtlscherer et al. confirmed the reduction of circulating miRNA 126 and miRNA 145 amongst others in the serum of patients with coronary artery disease [132]. Other miRNAs that qualify as biomarkers are miRNA 1, miRNA 133b and miRNA 499 which have been shown to be elevated in patients and animal models of acute myocardial infarction [133,134].

LncRNAs, non-coding RNAs with more than 200 nucleotides, are transcribed as overlapping sense and anti-sense transcripts to coding DNA regions regulating the transcription of corresponding overlapping mRNA [38]. Robb and colleagues found evidence for the regulation of eNOS expression by overlapping antisense lncRNA, [135] which was upregulated during hypoxia [136]. Also, hypoxia induces the antisense transcript to the $3^{\prime}$ UTR of HIF $-1 \alpha$ which is similarly expressed in renal cancer and different human tissues $[137,138]$.

Non-conding RNAs play a pivotal role in the pathogenesis of cardiovascular disease and offer the possibility to operate as diagnostic and prognostic biomarkers.

\section{Perspectives for treatment and prevention}

Unaltered epigenetic regulation pathways like DNA methylation, posttranslational chromatin modifications and ncRNAs provide the basis for healthy cardiovascular system. By now, many studies show positive effects and possibilities of treatment via epigenetic modulators. Based on these experiences therapeutic intervention of hypoxia associated diseases like asthma and chronic obstructive pulmonary disease as well as of inflammatory processes by modulation of epigenetic factors seems to be promising.

Nutritional factors are highly essential in the prevention of cardiovascular disease and comprise both, the avoidance of unfavourable food supplements such as high concentrations of low density lipoproteins and salt as well as the accentuation of beneficial effects of nutritional ingredients such as folic acid or resveratrol [139-143].

In 1993 Frankel et al. identified antioxidative effects for resveratrol $\left(3,4^{\prime}, 5\right.$-trihydroxystilbene) on low density lipoproteins (LDLs) thus reducing a crucial initiation event in the pathogenesis of atherosclerosis [144]. In addition, Wallerath et al. found evidence of resveratrol enhancing the activity and expression of eNOS in vitro [145]. In 2006, resveratrol, which is a plant derived activator of SIRT1, was found to decrease reactive oxygen species, inflammation, and apoptosis in the aortas of elderly mice that ran through a high-fat diet. Resveratrol is known to maintain normal endothelial function [146]. In transgenic apolipoprotein $\mathrm{E}$ null mice overexpression of SIRT1 was reported to reduce atherosclerosis due to increased endothelial cell survival and function [147]. In addition, SIRT1 was demonstrated to prevent excessive superoxide production and to reduce inflammation through inhibition of $\mathrm{NFKB}$ signalling and a reduction of ICAM-1 and VCAM-1 expression levels [148]. SIRT1 also induces and directly enhances the expression of eNOS, which decreases the speed of vascular senesce. The activation of SIRT1 therefore appears to be an important tool as to the therapy of cardiovascular disease. $[149,150]$ The regulation of SIRT3 is closely connected to SIRT1 and seems to protect against damage caused by myocardial infarction or chronic heart failure [151]. Resveratrol is a plant derived factor that is mainly found in berries, grape skin and red wine and was the first activator of SIRT1 to be identified [145]. Very recently it was shown in a mouse model that resveratrol prevents diet induced left ventricular hypertrophy as well as interstitial fibrosis and diastolic dysfunction \{Qin, 2012 $\# 16820$ \} . Resveratrol represents the tip of the iceberg when it comes to the effects of nutrition on the epigenome and thus, their role in the prevention and pathogenesis of cardiovascular disease.

Folates are part of the family of water-soluble B vitamins, which directly serve the one-carbon-metabolism with methionine for the production of S-adenosylmethionine. This in turn is the one-carbon-donor for the methylation of DNA [152]. Folic acid deficiency leads to global DNA 
hypomethylation which comes along with an increased risk for cancer and cardiovascular disease [70,139]. During the course of atherosclerosis smooth muscle cells transform and share a number of characteristic similarities with cancer cells such as the overexpression of proto-oncogenes, growth-factors as well as increased cell motility [142]. This transformation is based on the global hypomethylation of DNA. Accordingly, at the age of four weeks ApoE mice show changes in the DNA methylation pattern prior to the generation of atherosclerotic lesions [153]. Similarly, a reduction of $\sim 9 \%$ in C5-methylation was identified in advanced atherosclerotic plaques in humans [154]. Folic acid directly influences the function of vascular endothelial cells via improving NO production and vasodilation. Doshi et al. for example showed improvement of endothelial function in CVD patients after intervention with homocysteine \{Doshi, $2002 \# 16819\}$.

Resveratrol and B vitamins prove to be important both, in the prevention and the treatment of cardiovascular disease and carry the advantage of being practically free of side effects. Currently more than hundred clinical trials are ongoing or already completed studying the aspects of resveratrol or folate administration in subjects thus emphasizing their relevance for prevention of diet induced CVDs in humans (ClinicalTrials.gov).

In addition to nutritional factors a number of small molecule HDAC inhibitors for the treatment of cardiovascular disease are currently available:

In preclinical studies, most of the small molecule inhibitors of HDACS are hydroxamid acid derivates like the pan-HDAC inhibitor TSA and suberoylanilide hydroxamic acid (SAHA) [8].

HDAC inhibitors (HDACIs) are potential candidates in the treatment of cardiovascular disease such as cardiac hypertrophy, heart failure, ischemia/reperfusion injury, atherosclerosis and restenosis [155,156], Gallo et al. for example demonstrated in a mice model that the selective inhibition of class I HDACs with an apicidin derivate prevented cardiac hypertrophy and failure [157]. Rajasingh et al. treated mouse bone marrow progenitor cells with TSA and 5-Aza and further differentiated these cells into myocyte progenitors. The implantation of these cells led to an improvement of cardiac function after infarction. This points out another route small molecules unclose for CVD treatment \{Rajasingh, $2011 \# 16828\}$. In recent in vitro experiments TSA was shown to agonist load- and agonist-induced hypertrophy which suppressed autophagy of myocytes. These data where supported by the finding that the ventricular mass of hypertrophic animals was normalized as well as ventricular function \{Cao, 2011 \#16832\}.

In the treatment of AAA, there have recently been new results: Metacept-1 (MCT-1), a synthetic derivate of oxamflavin, also serves as an inhibitor of HDAC and showed AAA inhibitory effects in a mouse model [158]. HDAC inhibitors seem to constitute a potential way of managing AAA by targeting aortic proteolytic degradation.

\section{Conclusions}

In summary, class II and class III HDACs where shown to exert heart protective activities [95]. Further investigations are crucial to get a better understanding of the complex epigenetic interactions and to provide new ways for the treatment of vascular disease.

\section{Abbreviations}

AAA: Abdominal aortic aneurysm; CGRP: Calcitonin gene related peptide; DNMTs: DNA methyltransferases; ecSOD: Extracellular superoxide dismutase; EDN1: Plasma endothelin-1; eNOS: Synonym: NOS3, endothelial isoform of nitric oxide synthase; ESC: Embryonic stem cells; ET: Endothelin; GMCSF: Granulocyte macrophage colony-stimulating factor; HATs: Histone acetyltransferases; hCGBP: Human CpG binding protein; HDAC: Histone deacetylase; HDACls: HDAC inhibitors; HIF-1: Hypoxia-inducible factor; HIF1: Hypoxia-inducible factor 1-alpha; IUGR: Intra uterine growth restriction; lincRNAs: Large intervening non-coding RNAs; MEF2: myocyte enhancer factor-2; miRNA: microRNAs; MMP: Matrix metalloproteinase; ncRNA: Noncoding RNA; NO: Nitric oxide; ONOO-: Peroxynitrite; PAF: Plateletactivating factor; PRC2: Polycomb repressive complex 2; RISC: RNA-induced silencing complex; ROS: Reactive oxygen derivatives; SAHA: Suberoylanilide hydroxamic acid; siRNA: Short interfering RNAs; SMCs: Smooth muscle cells; SOCS: Suppressors of cytokine signalling; TGF- $B$ : Transforming growth factor beta; TSA: Trichostatin A; TXA2: Thromboxane; VEGF: Vascular endothelial growth factor; VSMCs: Vascular smooth muscle cells.

\section{Competing interests}

The authors declare that they have no competing interests.

\section{Authors' contributions}

All authors have written and edited the manuscript. All authors read and approved the final manuscript.

\section{Author details}

'Saarland University Medical Center, Department of Internal Medicine, Division of Immunotherapy and Gene Therapy, Homburg, Saar D-66421 Germany. ${ }^{2}$ Institute of Occupational and Social Medicine and Health Services Research, University of Tuebingen, Wilhelmstrasse 27, D-72074 Tuebingen, Germany.

Received: 3 January 2012 Accepted: 9 March 2012

Published: 23 May 2012

\section{References}

1. Matouk CC, Marsden PA: Epigenetic regulation of vascular endothelial gene expression. Circ Res 2008, 102(8):873-87.

2. Yan MS, Matouk CC, Marsden PA: Epigenetics of the vascular endothelium. J Appl Physiol 2010, 109(3):916-26.

3. Pons $D$, et al: Epigenetic histone acetylation modifiers in vascular remodelling: new targets for therapy in cardiovascular disease. Eur Heart J 2009, 30(3):266-77.

4. Krause B, Sobrevia L, Casanello P: Epigenetics: new concepts of old phenomena in vascular physiology. Curr Vasc Pharmacol 2009, 7(4):513-20.

5. Waddington CH: The epigenotype. Endeavour 1942, 1:18-20.

6. Goldberg AD, Allis CD, Bernstein E: Epigenetics: a landscape takes shape. Cell 2007, 128(4):635-8.

7. Taby R, Issa JP: Cancer epigenetics. CA Cancer J Clin 2010, 60(6):376-92.

8. Krishna SM, et al: Genetic and epigenetic mechanisms and their possible role in abdominal aortic aneurysm. Atherosclerosis 2010, 212(1):16-29.

9. Trojer $P$, Reinberg D: Facultative heterochromatin: is there a distinctive molecular signature? Mol Cell 2007, 28(1):1-13.

10. Bernstein $B E$, Meissner A, Lander ES: The mammalian epigenome. Cell 2007, 128(4):669-81. 
11. Miranda TB, Jones PA: DNA methylation: the nuts and bolts of repression. J Cell Physiol 2007, 213(2):384-90.

12. Cheng $X$, Blumenthal RM: Mammalian DNA methyltransferases: a structural perspective. Structure 2008, 16(3):341-50.

13. Schaefer $M$, et al: RNA methylation by Dnmt2 protects transfer RNAs against stress-induced cleavage. Genes Dev 2010, 24(15):1590-5.

14. Reik W: Stability and flexibility of epigenetic gene regulation in mammalian development. Nature 2007, 447(7143):425-32.

15. Goll MG, Bestor TH: Eukaryotic cytosine methyltransferases. Annu Rev Biochem 2005, 74:481-514.

16. Wu H, SUn YE: Epigenetic regulation of stem cell differentiation. Pediatr Res 2006, 59(4 Pt 2):21R-5R.

17. Bell AC, Felsenfeld G: Methylation of a CTCF-dependent boundary controls imprinted expression of the lgf2 gene. Nature 2000, 405(6785):482-5.

18. Comb M, Goodman HM: CpG methylation inhibits proenkephalin gene expression and binding of the transcription factor AP-2. Nucleic Acids Res 1990, 18(13):3975-82.

19. Harrington MA, et al: Cytosine methylation does not affect binding of transcription factor Sp1. Proc Natl Acad Sci U S A 1988, 85(7):2066-70.

20. Wenger $\mathrm{RH}$, et al: Oxygen-regulated erythropoietin gene expression is dependent on a CpG methylation-free hypoxia-inducible factor-1 DNAbinding site. Eur J Biochem 1998, 253(3):771-7.

21. Voo KS, et al: Cloning of a mammalian transcriptional activator that binds unmethylated CpG motifs and shares a CXXC domain with DNA methyltransferase, human trithorax, and methyl-CpG binding domain protein 1. Mol Cell Biol 2000, 20(6):2108-21.

22. Wang GG, Allis CD, Chi P: Chromatin remodeling and cancer, Part II: ATP-dependent chromatin remodeling. Trends Mol Med 2007, 13(9):373-80.

23. Schones DE, et al: Dynamic regulation of nucleosome positioning in the human genome. Cell 2008, 132(5):887-98.

24. Wang $L$, et al: Histone acetyltransferase activity is conserved between yeast and human GCN5 and is required for complementation of growth and transcriptional activation. Mol Cell Biol 1997, 17(1):519-27.

25. Shahbazian MD, Grunstein M: Functions of site-specific histone acetylation and deacetylation. Annu Rev Biochem 2007, 76:75-100.

26. Shogren-Knaak M, et al: Histone H4-K16 acetylation controls chromatin structure and protein interactions. Science 2006, 311(5762):844-7.

27. Zschoernig B, Mahlknecht U: SIRTUIN 1: regulating the regulator. Biochem Biophys Res Commun 2008, 376(2):251-5.

28. Voelter-Mahlknecht $\mathrm{S}$, Mahlknecht $\mathrm{U}$ : The sirtuins in the pathogenesis of cancer. Clinical Epigenetics 2010, 1(3-4):71-83.

29. Shi $X$, et al: Proteome-wide analysis in Saccharomyces cerevisiae identifies several PHD fingers as novel direct and selective binding modules of histone $\mathrm{H} 3$ methylated at either lysine 4 or lysine 36 . J Biol Chem 2007, 282(4):2450-5.

30. Chen W, Bacanamwo M, Harrison DG: Activation of p300 histone acetyltransferase activity is an early endothelial response to laminar shear stress and is essential for stimulation of endothelial nitric-oxide synthase mRNA transcription. J Biol Chem 2008, 283(24):16293-8.

31. Heintzman ND, et al: Distinct and predictive chromatin signatures of transcriptional promoters and enhancers in the human genome. Nat Genet 2007, 39(3):311-8.

32. Barski $A$, et al: High-resolution profiling of histone methylations in the human genome. Cell 2007, 129(4):823-37.

33. Zaratiegui M, Irvine DV, Martienssen RA: Noncoding RNAs and gene silencing. Cell 2007, 128(4):763-76.

34. Noma K, et al: RITS acts in cis to promote RNA interference-mediated transcriptional and post-transcriptional silencing. Nat Genet 2004, 36(11):1174-80.

35. Mercer TR, Dinger ME, Mattick JS: Long non-coding RNAs: insights into functions. Nat Rev Genet 2009, 10(3):155-9.

36. Khalil AM, et al: Many human large intergenic noncoding RNAs associate with chromatin-modifying complexes and affect gene expression. Proc Natl Acad Sci U S A 2009, 106(28):11667-72.

37. Morris KV: siRNA-mediated transcriptional gene silencing: the potential mechanism and a possible role in the histone code. Cell Mol Life Sci 2005, 62(24):3057-66.
38. Kaikkonen MU, Lam MT, Glass CK: Non-coding RNAs as regulators of gene expression and epigenetics. Cardiovasc Res 2011, 90(3):430-40.

39. Lee $Y$, et al: The nuclear RNase III Drosha initiates microRNA processing. Nature 2003, 425(6956):415-9.

40. Denli AM, et al: Processing of primary microRNAs by the Microprocessor complex. Nature 2004, 432(7014):231-5.

41. Bavan L, Midwood K, Nanchahal J: MicroRNA epigenetics: a new avenue for wound healing research. BioDrugs 2011, 25(1):27-41.

42. Morris KV, et al: Small interfering RNA-induced transcriptional gene silencing in human cells. Science 2004, 305(5688):1289-92.

43. Ting AH, et al: Short double-stranded RNA induces transcriptional gene silencing in human cancer cells in the absence of DNA methylation. Nat Genet 2005, 37(8):906-10.

44. Risau W: Mechanisms of angiogenesis. Nature 1997, 386(6626):671-4.

45. Kubis N, Levy Bl: Understanding angiogenesis: a clue for understanding vascular malformations. J Neuroradiol 2004, 31(5):365-8.

46. Hamik A, Wang B, Jain MK: Transcriptional regulators of angiogenesis. Arterioscler Thromb Vasc Biol 2006, 26(9):1936-47.

47. Guarani $V$, et al: Acetylation-dependent regulation of endothelial Notch signalling by the SIRT1 deacetylase. Nature 2011, 473(7346):234-8.

48. Jones EA, le Noble F, Eichmann A: What determines blood vesse structure? Genetic prespecification vs. hemodynamics. Physiology (Bethesda) 2006, 21:388-95.

49. Hirashima M, Suda T: Differentiation of arterial and venous endothelia cells and vascular morphogenesis. Endothelium 2006, 13(2):137-45.

50. le Noble F, et al: Neural guidance molecules, tip cells, and mechanical factors in vascular development. Cardiovasc Res 2008, 78(2):232-41.

51. McDonald OG, Owens GK: Programming smooth muscle plasticity with chromatin dynamics. Circ Res 2007, 100(10):1428-41.

52. Rossig L, et al: Inhibitors of histone deacetylation downregulate the expression of endothelial nitric oxide synthase and compromise endothelial cell function in vasorelaxation and angiogenesis. Circ Res 2002, 91(9):837-44.

53. Chan $Y$, et al: The cell-specific expression of endothelial nitric-oxide synthase: a role for DNA methylation. J Biol Chem 2004, 279(33):35087-100.

54. Fish JE, et al: The expression of endothelial nitric-oxide synthase is controlled by a cell-specific histone code. J Biol Chem 2005, 280(26):24824-38.

55. Fish JE, Marsden PA: Endothelial nitric oxide synthase: insight into cellspecific gene regulation in the vascular endothelium. Cell Mol Life Sci 2006, 63(2):144-62.

56. Kim MS, et al: Histone deacetylases induce angiogenesis by negative regulation of tumor suppressor genes. Nat Med 2001, 7(4):437-43.

57. Zeng $L$, et al: HDAC3 is crucial in shear- and VEGF-induced stem cell differentiation toward endothelial cells. J Cell Biol 2006, 174(7):1059-69.

58. Illi $B$, et al: Epigenetic histone modification and cardiovascular lineage programming in mouse embryonic stem cells exposed to laminar shear stress. Circ Res 2005, 96(5):501-8.

59. Deroanne CF, et al: Histone deacetylases inhibitors as anti-angiogenic agents altering vascular endothelial growth factor signaling. Oncogene 2002, 21(3):427-36

60. Chang $S$, et al: Histone deacetylase 7 maintains vascular integrity by repressing matrix metalloproteinase 10 . Cell 2006, 126(2):321-34.

61. Wang $S$, et al: Control of endothelial cell proliferation and migration by VEGF signaling to histone deacetylase 7. Proc Natl Acad Sci U S A 2008, 105(22):7738-43.

62. Xiao Q, et al: Sca-1+ progenitors derived from embryonic stem cells differentiate into endothelial cells capable of vascular repair after arterial injury. Arterioscler Thromb Vasc Biol 2006, 26(10):2244-51.

63. Rossig $\mathrm{L}$, et al: Histone deacetylase activity is essential for the expression of HoxA9 and for endothelial commitment of progenitor cells. J Exp Med 2005, 201(11):1825-35.

64. Diehl F, et al: The histone methyltransferase MLL is an upstream regulator of endothelial-cell sprout formation. Blood 2007, 109(4):1472-8.

65. Van Speybroeck L: From epigenesis to epigenetics: the case of C. $\mathrm{H}$. Waddington. Ann N Y Acad Sci 2002, 981:61-81.

66. Jirtle RL, Skinner MK: Environmental epigenomics and disease susceptibility. Nat Rev Genet 2007, 8(4):253-62.

67. Escudero C, Sobrevia L: A hypothesis for preeclampsia: adenosine and inducible nitric oxide synthase in human placental microvascular endothelium. Placenta 2008, 29(6):469-83. 
68. Casanello P, Escudero C, Sobrevia L: Equilibrative nucleoside (ENTs) and cationic amino acid (CATs) transporters: implications in foetal endothelial dysfunction in human pregnancy diseases. Curr Vasc Pharmacol 2007, 5 (1):69-84.

69. Hellebrekers DM, Griffioen AW, van Engeland M: Dual targeting of epigenetic therapy in cancer. Biochim Biophys Acta 2007, 1775(1):76-91.

70. Kim $\mathrm{Yl}$, et al: Folate deficiency in rats induces DNA strand breaks and hypomethylation within the p53 tumor suppressor gene. Am J Clin Nutr 1997, 65(1):46-52.

71. Mottet $D$, et al: Histone deacetylase 7 silencing alters endothelial cell migration, a key step in angiogenesis. Circ Res 2007, 101(12):1237-46.

72. Potente $M$, et al: SIRT1 controls endothelial angiogenic functions during vascular growth. Genes Dev 2007, 21(20):2644-58.

73. Brunet $A$, et al: Stress-dependent regulation of FOXO transcription factors by the SIRT1 deacetylase. Science 2004, 303(5666):2011-5.

74. Yamamoto $\mathrm{K}$, et al: Fluid shear stress induces differentiation of Flk-1positive embryonic stem cells into vascular endothelial cells in vitro. Am J Physiol Heart Circ Physiol 2005, 288(4):H1915-24.

75. Illi $\mathrm{B}$, et al: Shear stress-mediated chromatin remodeling provides molecular basis for flow-dependent regulation of gene expression. Circ Res 2003, 93(2):155-61.

76. Johnson $A B$, Denko N, Barton MC: Hypoxia induces a novel signature of chromatin modifications and global repression of transcription. Mutat Res 2008, 640(1-2):174-9.

77. Johnson $A B$, Barton MC: Hypoxia-induced and stress-specific changes in chromatin structure and function. Mutat Res 2007, 618(1-2):149-62

78. Fish JE, et al: Hypoxic repression of endothelial nitric-oxide synthase transcription is coupled with eviction of promoter histones. J Biol Chem 2010, 285(2):810-26.

79. Wang $Y$, et al: Regulation of endocytosis via the oxygen-sensing pathway. Nat Med 2009, 15(3):319-24

80. Kato H, Tamamizu-Kato S, Shibasaki F: Histone deacetylase 7 associates with hypoxia-inducible factor 1 alpha and increases transcriptional activity. J Biol Chem 2004, 279(40):41966-74

81. Monraats PS, et al: Genetic inflammatory factors predict restenosis after percutaneous coronary interventions. Circulation 2005, 112(16):2417-25.

82. Libby P, Ridker PM, Maseri A: Inflammation and atherosclerosis. Circulation 2002, 105(9):1135-43.

83. Mertens G: Gene/Environment interaction in atherosclerosis: an example of clinical medicine as seen from the evolutionary perspective. Int $J$ Hypertens 2010, 2010:654078.

84. Stenvinkel $P$, et al: Impact of inflammation on epigenetic DNA methylation - a novel risk factor for cardiovascular disease? J Intern Med 2007, 261(5):488-99

85. Galm O, et al: SOCS-1, a negative regulator of cytokine signaling, is frequently silenced by methylation in multiple myeloma. Blood 2003, 101 (7):2784-8.

86. Chiba T, et al: A selective NFkappaB inhibitor, DHMEQ, reduced atherosclerosis in ApoE-deficient mice. J Atheroscler Thromb 2006, 13 (6):308-13.

87. Ito $\mathrm{K}$, et al: Oxidative stress reduces histone deacetylase 2 activity and enhances IL- 8 gene expression: role of tyrosine nitration. Biochem Biophys Res Commun 2004, 315(1):240-5.

88. Nie M, Knox AJ, Pang L: beta2-Adrenoceptor agonists, like glucocorticoids, repress eotaxin gene transcription by selective inhibition of histone $\mathrm{H} 4$ acetylation. J Immunol 2005, 175(1):478-86.

89. Haley $\mathrm{KJ}$, et al: Overexpression of eotaxin and the CCR3 receptor in human atherosclerosis: using genomic technology to identify a potential novel pathway of vascular inflammation. Circulation 2000 102(18):2185-9.

90. Zhang XH, Ma XJ, Zhao T: Effects of granulocyte-macrophage colony stimulating factor on the repair of vessel intima damaged by balloon. Chin Med J (Engl) 2005, 118(3):220-5.

91. Tomita K, Barnes PJ, Adcock IM: The effect of oxidative stress on histone acetylation and IL-8 release. Biochem Biophys Res Commun 2003, 301 (2):572-7.

92. Ito K, Barnes PJ, Adcock IM: Glucocorticoid receptor recruitment of histone deacetylase 2 inhibits interleukin-1beta-induced histone $\mathrm{H} 4$ acetylation on lysines 8 and 12. Mol Cell Biol 2000, 20(18):6891-903.
93. Cosio $B G$, et al: Theophylline restores histone deacetylase activity and steroid responses in COPD macrophages. J Exp Med 2004, 200(5):689-95.

94. Granger A, et al: Histone deacetylase inhibition reduces myocardial ischemia-reperfusion injury in mice. FASEB J 2008, 22(10):3549-60.

95. McKinsey $T A$, Zhang $C L$, Olson EN: Signaling chromatin to make muscle. Curr Opin Cell Biol 2002, 14(6):763-72.

96. Zhang $\mathrm{CL}$, et al: Class II histone deacetylases act as signal-responsive repressors of cardiac hypertrophy. Cell 2002, 110(4):479-88.

97. Henderson EL, et al: Death of smooth muscle cells and expression of mediators of apoptosis by T lymphocytes in human abdominal aortic aneurysms. Circulation 1999, 99(1):96-104.

98. Longo GM, et al: Matrix metalloproteinases 2 and 9 work in concert to produce aortic aneurysms. J Clin Invest 2002, 110(5):625-32.

99. Sakalihasan N, et al: Activated forms of MMP2 and MMP9 in abdominal aortic aneurysms. J Vasc Surg 1996, 24(1):127-33.

100. Kowalewski R, et al: Evaluation of enzymes involved in proteoglycan degradation in the wall of abdominal aortic aneurysms. J Vasc Res 2006, 43(1):95-100.

101. Raynaud M, Barlow T: On local asphyxia and symmetrical gangrene of the extremities. The Sydenham Society: London; 1888.

102. Chang K, et al: The Glu298Asp polymorphism in the endothelial nitric oxide synthase gene is strongly associated with coronary spasm. Coron Artery Dis 2003, 14(4):293-9.

103. Baek SJ, Lee KD, Shen RF: Genomic structure and polymorphism of the human thromboxane synthase-encoding gene. Gene 1996, 173(2):251-6.

104. Amano S, et al: Polymorphism of the promoter region of prostacyclin synthase gene in chronic thromboembolic pulmonary hypertension. Respirology 2004, 9(2):184-9.

105. Vallance $\mathrm{P}$, Collier J, Moncada S: Effects of endothelium-derived nitric oxide on peripheral arteriolar tone in man. Lancet 1989, 2(8670):997-1000.

106. Kennedy G, et al: Endothelial activation and response in patients with hand arm vibration syndrome. Eur J Clin Invest 1999, 29(7):577-81.

107. Herrick AL: Pathogenesis of Raynaud's phenomenon. Rheumatology (Oxford) 2005, 44(5):587-96.

108. Radomski MW, Palmer RM, Moncada S: The anti-aggregating properties of vascular endothelium: interactions between prostacyclin and nitric oxide. Br J Pharmacol 1987, 92(3):639-46.

109. Liapina M, Tzvetkov D, Vodenitcharov E: Pathophysiology of vibrationinduced white fingers-current opinion: a review. Cent Eur J Public Health 2002, 10(1-2):16-20.

110. Cines DB, et al: Endothelial cells in physiology and in the pathophysiology of vascular disorders. Blood 1998, 91(10):3527-61.

111. Tai SC, Robb GB, Marsden PA: Endothelial nitric oxide synthase: a new paradigm for gene regulation in the injured blood vessel. Arterioscler Thromb Vasc Biol 2004, 24(3):405-12.

112. Laufs U, Liao JK: Post-transcriptional regulation of endothelial nitric oxide synthase mRNA stability by Rho GTPase. J Bio/ Chem 1998, 273(37):24266-71.

113. Bouloumie A, Schini-Kerth VB, Busse R: Vascular endothelial growth factor up-regulates nitric oxide synthase expression in endothelial cells. Cardiovasc Res 1999, 41(3):773-80

114. Davis ME, et al: Shear stress regulates endothelial nitric oxide synthase expression through c-Src by divergent signaling pathways. Circ Res 2001, 89(11):1073-80.

115. Yoshizumi $M$, et al: Tumor necrosis factor downregulates an endothelial nitric oxide synthase mRNA by shortening its half-life. Circ Res 1993, 73 (1):205-9.

116. Liao JK, et al: Oxidized low-density lipoprotein decreases the expression of endothelial nitric oxide synthase. J Biol Chem 1995, 270(1):319-24.

117. Gryglewski RJ, Palmer RM, Moncada S: Superoxide anion is involved in the breakdown of endothelium-derived vascular relaxing factor. Nature 1986, 320(6061):454-6.

118. Garg UC, Hassid A: Nitric oxide-generating vasodilators and 8-bromo-cyclic guanosine monophosphate inhibit mitogenesis and proliferation of cultured rat vascular smooth muscle cells. J Clin Invest 1989, 83(5):1774-7.

119. Beckman JS, et al: Apparent hydroxyl radical production by peroxynitrite: implications for endothelial injury from nitric oxide and superoxide. Proc Natl Acad Sci U S A 1990, 87(4):1620-4.

120. Rubbo H, Denicola A, Radi R: Peroxynitrite inactivates thiol-containing enzymes of Trypanosoma cruzi energetic metabolism and inhibits cell respiration. Arch Biochem Biophys 1994, 308(1):96-102. 
121. Radi R, et al: Peroxynitrite oxidation of sulfhydryls. The cytotoxic potential of superoxide and nitric oxide. J Biol Chem 1991, 266(7):4244-50.

122. Radi $R$, et al: Peroxynitrite-induced membrane lipid peroxidation: the cytotoxic potential of superoxide and nitric oxide. Arch Biochem Biophys 1991, 288(2):481-7.

123. Munzel T, et al: Role for NADPH/NADH oxidase in the modulation of vascular tone. Ann N Y Acad Sci 1999, 874:386-400.

124. Liu S, Beckman JS, Ku DD: Peroxynitrite, a product of superoxide and nitric oxide, produces coronary vasorelaxation in dogs. J Pharmacol Exp Ther 1994, 268(3):1114-21.

125. Forstermann $U$, Munzel $T$ : Endothelial nitric oxide synthase in vascular disease: from marvel to menace. Circulation 2006, 113(13):1708-14.

126. Ji $R$, et al: MicroRNA expression signature and antisense-mediated depletion reveal an essential role of MicroRNA in vascular neointimal lesion formation. Circ Res 2007, 100(11):1579-88,

127. Liu X, et al: MicroRNA-222 regulates cell invasion by targeting matrix metalloproteinase 1 (MMP1) and manganese superoxide dismutase 2 (SOD2) in tongue squamous cell carcinoma cell lines. Cancer Genomics Proteomics 2009, 6(3):131-9.

128. Zernecke A, et al: Delivery of microRNA-126 by apoptotic bodies induces CXCL12-dependent vascular protection. Sci Signal 2009, 100:ra81.

129. Harris TA, et al: MicroRNA-126 regulates endothelial expression of vascular cell adhesion molecule 1. Proc Natl Acad Sci U S A 2008, 105 (5):1516-21.

130. Menghini $R$, et al: MicroRNA 217 modulates endothelial cell senescence via silent information regulator 1. Circulation 2009, 120(15):1524-32.

131. Dimmeler S, Zeiher AM: Circulating microRNAs: novel biomarkers for cardiovascular diseases? Eur Heart J 2010, 31(22):2705-7.

132. Fichtlscherer $S$, et al: Circulating microRNAs in patients with coronary artery disease. Circ Res 2010, 107(5):677-84.

133. Wang R, et al: Circulating MicroRNAs are Promising Novel Biomarkers of Acute Myocardial Infarction. Intern Med 2011, 50(17):1789-95.

134. D'Alessandra $Y$, et al: Circulating microRNAs are new and sensitive biomarkers of myocardial infarction. Eur Heart J 2010, 31(22):2765-73.

135. Robb GB, et al: Post-transcriptional regulation of endothelial nitric-oxide synthase by an overlapping antisense mRNA transcript. J Biol Chem 2004, 279(36):37982-96

136. Fish JE, et al: Hypoxia-inducible expression of a natural cis-antisense transcript inhibits endothelial nitric-oxide synthase. J Biol Chem 2007, 282 (21):15652-66.

137. Thrash-Bingham CA, Tartof KD: aHIF-1: a natural antisense transcript overexpressed in human renal cancer and during hypoxia. $J$ Natl Cancer Inst 1999, 91(2):143-51.

138. Rossignol F, Vache C, Clottes E: Natural antisense transcripts of hypoxiainducible factor 1alpha are detected in different normal and tumour human tissues. Gene 2002, 299(1-2):135-40.

139. McNulty H, et al: Homocysteine, B-vitamins and CVD. Proc Nutr Soc 2008 67(2):232-7

140. Farley TA, et al: Deaths preventable in the U.S. by improvements in use of clinical preventive services. Am J Prev Med 2010, 38(6):600-9.

141. Duthie SJ, et al: Blood folate status and expression of proteins involved in immune function, inflammation, and coagulation: biochemical and proteomic changes in the plasma of humans in response to long-term synthetic folic acid supplementation. J Proteome Res 2010, 9(4):1941-50.

142. Duthie SJ: Epigenetic modifications and human pathologies: cancer and CVD. Proc Nutr Soc 2011, 70(1):47-56.

143. Das M, Das DK: Resveratrol and cardiovascular health. Mol Aspects Med 2010, 31(6):503-12.

144. Frankel EN, Waterhouse AL, Kinsella JE: Inhibition of human LDL oxidation by resveratrol. Lancet 1993, 341(8852):1103-4.

145. Wallerath $T$, et al: Resveratrol, a polyphenolic phytoalexin present in red wine, enhances expression and activity of endothelial nitric oxide synthase. Circulation 2002, 106(13):1652-8.

146. Lagouge $M$, et al: Resveratrol improves mitochondrial function and protects against metabolic disease by activating SIRT1 and PGC-1alpha. Cell 2006, 127(6):1109-22.

147. Zhang QJ, et al: Endothelium-specific overexpression of class III deacetylase SIRT1 decreases atherosclerosis in apolipoprotein E-deficient mice. Cardiovasc Res 2008, 80(2):191-9.

148. Stein S, et al: SIRT1 reduces endothelial activation without affecting vascular function in ApoE-/- mice. Aging (Albany NY) 2010, 2(6):353-60.
149. Ota $\mathrm{H}$, et al: Sirt1 modulates premature senescence-like phenotype in human endothelial cells. J Mol Cell Cardiol 2007, 43(5):571-9.

150. Mattagajasingh I, et al: SIRT1 promotes endothelium-dependent vascular relaxation by activating endothelial nitric oxide synthase. Proc Natl Acad Sci U S A 2007, 104(37):14855-60.

151. Haigis MC, Sinclair DA: Mammalian sirtuins: biological insights and disease relevance. Annu Rev Pathol 2010, 5:253-95.

152. Suzuki $H$, et al: DNA methylation and cancer pathways in gastrointestinal tumors. Pharmacogenomics 2008, 9(12):1917-28.

153. Lund $G$, et al: DNA methylation polymorphisms precede any histological sign of atherosclerosis in mice lacking apolipoprotein E. J Biol Chem 2004, 279(28):29147-54.

154. Hiltunen $\mathrm{MO}$, et al: DNA hypomethylation and methyltransferase expression in atherosclerotic lesions. Vasc Med 2002, 7(1):5-11.

155. Van Wagoner DR, Nattel S: Insights into mechanisms linking cardiac hypertrophy and atrial fibrosis: evidence for a role of histone deacetylase in atrial fibrillation pathophysiology and therapy. J Mol Cell Cardiol 2008 45(6):707-8.

156. Pons D, Jukema JW: Epigenetic histone acetylation modifiers in vascular remodelling - new targets for therapy in cardiovascular disease. Neth Heart J 2008, 16(1):30-2.

157. Gallo P, et al: Inhibition of class I histone deacetylase with an apicidin derivative prevents cardiac hypertrophy and failure. Cardiovasc Res 2008 80(3):416-24.

158. Vinh A, et al: A novel histone deacetylase inhibitor reduces abdominal aortic aneurysm formation in angiotensin II-infused apolipoprotein Edeficient mice. J Vasc Res 2008, 45(2):143-52.

doi:10.1186/1868-7083-4-7

Cite this article as: Schleithoff et al:: On the epigenetics of vascular regulation and disease. Clinical Epigenetics 2012 4:7.

\section{Submit your next manuscript to BioMed Central and take full advantage of:}

- Convenient online submission

- Thorough peer review

- No space constraints or color figure charges

- Immediate publication on acceptance

- Inclusion in PubMed, CAS, Scopus and Google Scholar

- Research which is freely available for redistribution
C) BioMed Central 